\title{
Biopsy-proven lymphocytic myocarditis following first mRNA COVID-19 vaccination in a 40-year-old male: case report
}

\author{
Peter Ehrlich ${ }^{1}$ Karin Klingel ${ }^{2}$. Susanne OhImann-Knafo ${ }^{3} \cdot$ Sebastian Hüttinger $^{3} \cdot$ Nitin Sood $^{1}$ - Dirk Pickuth ${ }^{3}$. \\ Michael Kindermann ${ }^{1}[$
}

Received: 8 July 2021 / Accepted: 30 August 2021 / Published online: 6 September 2021

(c) Springer-Verlag GmbH Germany, part of Springer Nature 2021

Sirs:

We report a 40-year-old, previously healthy man who was admitted to our emergency unit by his family doctor because of fever (till $39^{\circ} \mathrm{C}$ ), headache, chest pain and shortness of breath. Six days ago, he got the first dose of the mRNA COVID-19 vaccine Comirnaty ${ }^{\circledR}$ (BioNTech/Pfizer). Two days after vaccination, he developed fever and headache and another 2 days later, he suffered from resting dyspnea and angina pectoris.

Physical examination on admission revealed no pathologies. The first resting electrocardiogram (ECG 1) showed sinus rhythm with 82 beats per minute without any signs of acute ischemia (Fig. 1). Laboratory tests showed a highly increased high-sensitive Troponin $\mathrm{T}$ concentration of $952 \mathrm{ng} / \mathrm{l}$ (rising to $1030 \mathrm{ng} / \mathrm{l}$ after three hours; normal range $<15 \mathrm{ng} / \mathrm{l})$, increased concentrations of creatine kinase (CK) (666 U/l, rising to $763 \mathrm{U} / 1$ three hours later; normal range $<190 \mathrm{U} / \mathrm{l})$ and CK-MB isoenzyme (68 U/l; $75 \mathrm{U} / \mathrm{l}$ after three hours; normal range $<25 \mathrm{U} / \mathrm{l})$, an increased D-dimer level $(0.87 \mathrm{mg} / \mathrm{l}$; normal range $<0.55 \mathrm{mg} / \mathrm{l})$ and an elevated C-reactive protein $(50.9 \mathrm{mg} / \mathrm{l}$; normal range $<5 \mathrm{mg} / \mathrm{l})$ without leukocytosis; creatinine clearance, hemoglobin concentration, eosinophilic and platelet count and liver enzymes

Michael Kindermann

m.kindermann@caritasklinikum.de

1 Department of Cardiology, Angiology and Pneumology, CaritasKlinikum St. Theresia, Academic Teaching Hospital of the Saarland University, Rheinstr. 2, 66113 Saarbrücken, Germany

2 Cardiopathology, Institute for Pathology and Neuropathology, University Hospital Tübingen, Liebermeisterstr. 8, 72076 Tübingen, Germany

3 Department of Diagnostic and Interventional Radiology, CaritasKlinikum St. Theresia, Academic Teaching Hospital of the Saarland University, Rheinstr. 2, 66113 Saarbrücken, Germany were all normal. Blood cultures remained sterile. The SARSCoV-2 PCR of a nasopharyngeal swap was negative as well as SARS-CoV-2 antibody testing ( $\operatorname{IgG}$ and $\operatorname{IgA}$ ).

Pulmonary embolism was ruled out by CT angiography and sinus vein thrombosis was excluded by a cranial MRI scan.

Transthoracic echocardiograpy in the emergency unit showed a normally sized left ventricle (end-diastolic diameter $52 \mathrm{~mm}$ ) with a moderately reduced ejection fraction of $45 \%$ (biplane Simpson method) without regional wall motion abnormalities. The thickness of the basal septum was slightly increased to $13 \mathrm{~mm}$. Pericardial effusion could be ruled out as well as a valvular heart disease.

The patient was transferred to the intermediate care unit with the working diagnosis of "non-ST segment elevation myocardial infarction (NSTEMI)" versus "myocarditis", and therapy with acetylsalicylic acid, unfractionated heparin, an ACE inhibitor, a beta-blocker and a mineralocorticoid antagonist was started.

On the next morning, chest pain worsened and ECG showed ST elevations in leads I, II, aVL, V5-V6 and ST depression in leads III and aVR (ECG 2 in Fig. 2), which prompted emergency coronary angiography using transradial access. As no coronary artery disease could be diagnosed and laevo-cardiography showed a globally reduced ejection fraction of $43 \%$ (see supplementary data file), left ventricular endomyocardial biopsy was performed to exclude a potentially life-threatening type of myocarditis. Histology and immuno-histology of the biopsies revealed acute lymphocytic myocarditis (Fig. 3). (RT-)PCRs for common cardiotropic viruses or bacteria were negative.

Conservative heart failure therapy achieved complete remission of all symptoms within two days. Left ventricular ejection fraction normalized to $60 \%$ forty-eight hours after admission. On day four after admission, the patient was discharged from hospital free of symptoms. Heart failure medication was continued. He was advised to refrain from 
Fig. 1 First ECG of the patient immediately after admission to the emergency room
Fig. 2 ECG $15 \mathrm{~h}$ after admission with new ST elevations in leads I, II, aVL, V5-V6 and ST depression in leads III and aVR accompanied by increasing chest pain

\section{ECG $1(10 \mathrm{~mm} / \mathrm{mV}, 50 \mathrm{~mm} / \mathrm{s})$}
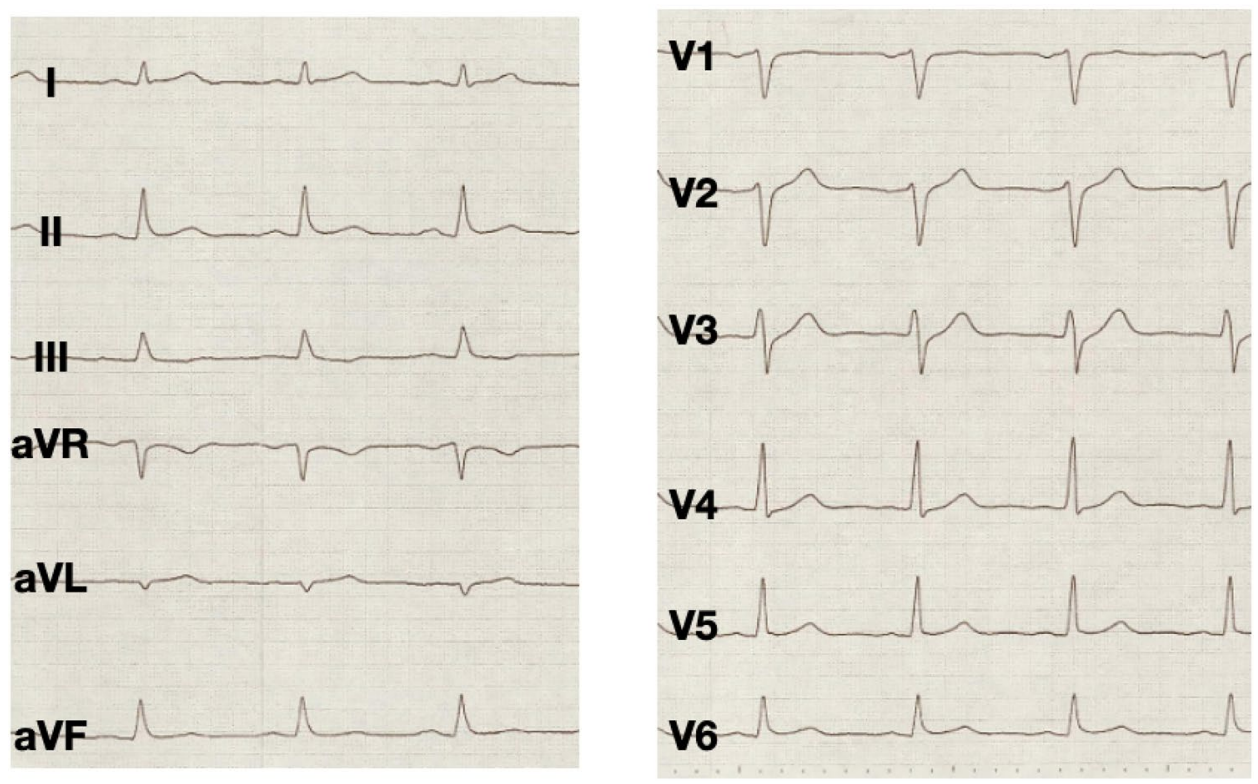

\section{ECG $2(10 \mathrm{~mm} / \mathrm{mv}, 50 \mathrm{~mm} / \mathrm{s})$}

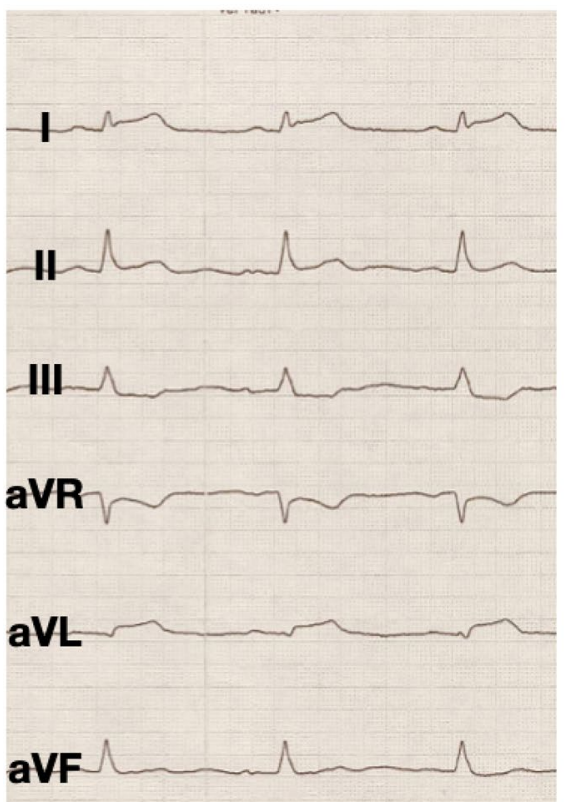

V1
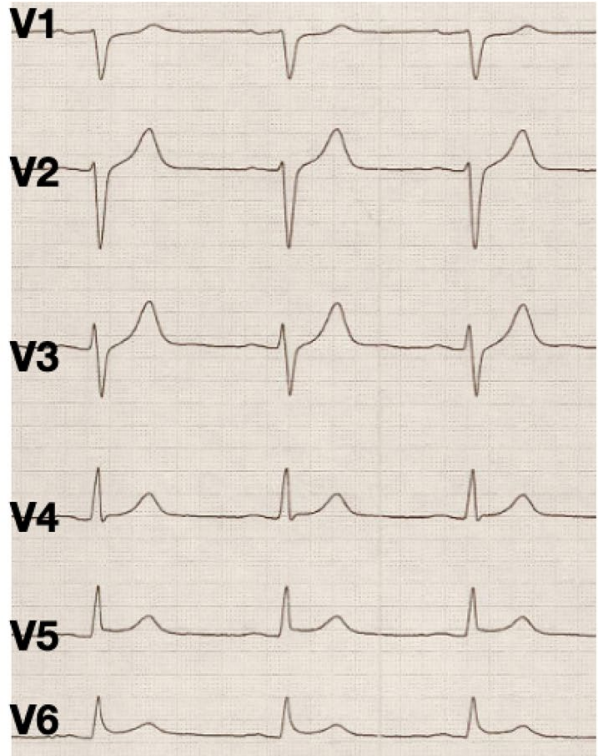

high-intense physical activity in the upcoming 3 months until cardiological follow-up.

A cardiac MRI study was carried out post-inpatient on day eight after admission. Cine images confirmed a normalized left ventricular ejection fraction $(69 \%)$ without regional wall motion abnormalities. However, it revealed increased left ventricular wall thickness with a septal thickness of $16 \mathrm{~mm}$ at maximum and a persistent myocardial inflammation throughout the left ventricle: myocardial hyper-intensities on $\mathrm{T} 2 \mathrm{w}$ images indicating myocardial edema were detected in the left ventricle, primarily in the basal and mid inferoseptal and anterolateral segments as well as in the apical lateral segment. Diffuse late gadolinium enhancement was noted in the basal and mid anteroseptal and inferoseptal segments as well as in the apical septal segment. Focal enhancement was mainly seen in the basal and mid anterolateral segments, in the apical lateral segment as well as in the apical cap. Approximately $90 \%$ of the total 
Fig. 3 Endomyocardial biopsy reveals areas of inflammation and myocyte necrosis (arrows in $\mathbf{A}$, Giemsa stain). The majority of immune cells represent CD68-positive macrophages (B) but also numerous CD3-positive $\mathrm{T}$ cells $(\mathbf{C})$ are present in the interstitium

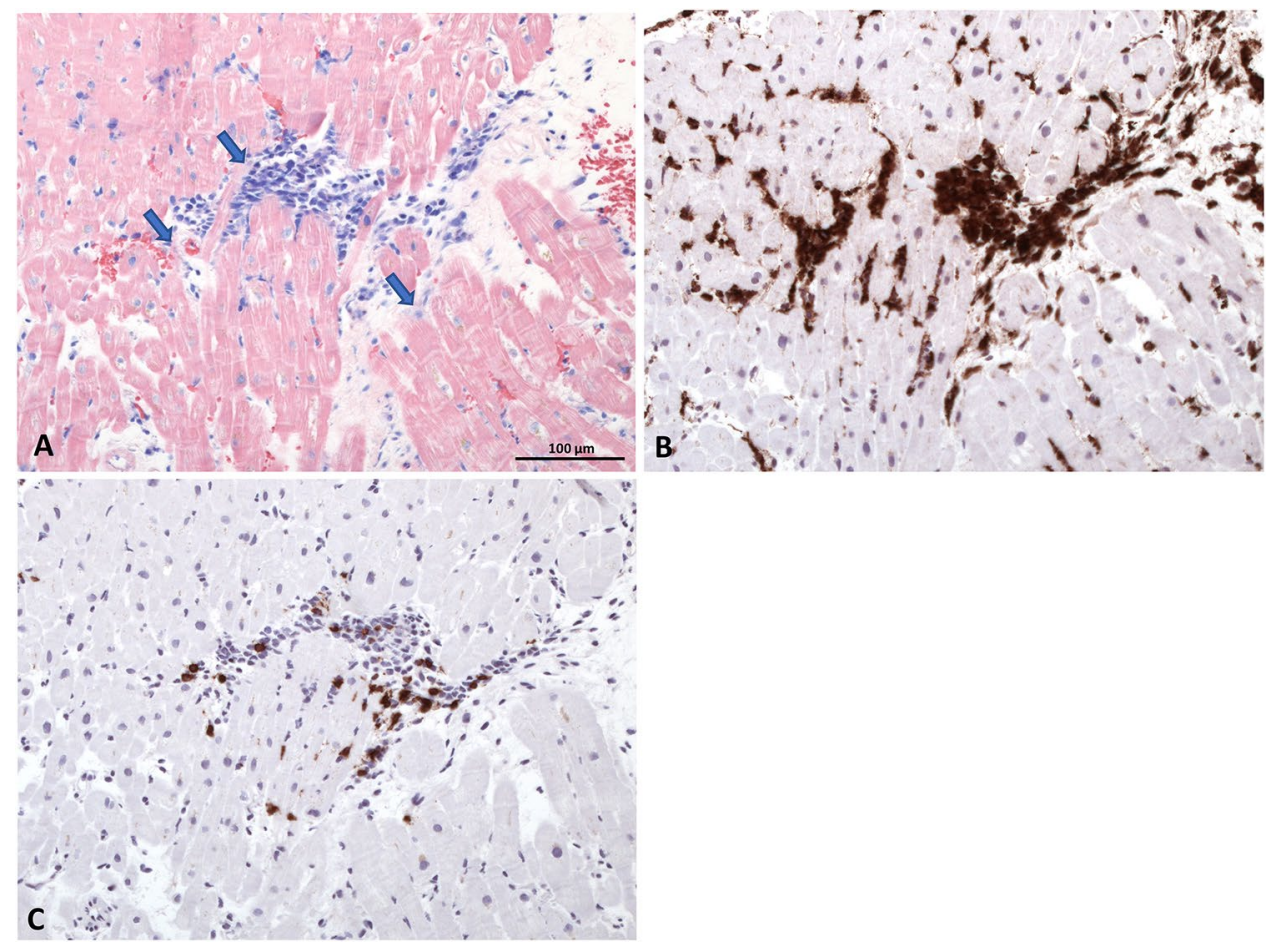

left ventricular mass was affected. There was no increased pericardial enhancement. Likewise, no pericardial effusion was evident (Fig. 4). A follow-up cardiac MRI study will be scheduled three months after hospital discharge.

The first reports of a small number of cases of suspected myocarditis after administration of the BioNTech/Pfizer COVID-19 vaccine came from Israel. A total of 275 cases of myocarditis were reported in Israel between December 2020 and May 2021 among more than five million vaccinated people. More than four-fifth of these cases occurred after the second vaccination dose and most cases were reported among men aged 16-19 years who had a mild clinical course with a hospital stay no longer than four days [2].

The US American CDC data (thru June 11, 2021) showed that after 5.8 million first doses of an mRNA COVID-19 vaccine administered to men aged 18-24 there were 47 reports of myocarditis or pericarditis, when three to 34 would have been expected. After the second vaccination dose, 233 cases were reported among 4.3 million vaccinated men, while two to 25 cases of myocarditis or pericarditis would have been expected. For females, the reported event rates after the first mRNA vaccination dose were within the expected event range for all age groups. But after the second vaccination dose, the number of reported myocarditis/ pericarditis cases in females aged 18-24 years went up to 27, slightly exceeding the rate in the general population which is two to 18 cases [3]. The US American Vaccine Safety Datalink (VSD) has repeatedly calculated the relative risk for developing myocarditis/pericarditis according to the
CDC case definition after mRNA COVID-19 vaccination compared with individuals who got any other vaccination on the same calendar days. While the overall adjusted risk ratio for myocarditis/pericarditis after either dose of any mRNA COVID-19 vaccine was not significantly increased at 1.07 (95\% CI 0.70-1.67; data from June 5, 2021), this looks different for the young: for 12-39-year-old individuals, the risk of getting myocarditis or pericarditis within 21 days after any of both doses of an mRNA COVID-19 vaccine was 3.5 times higher (95\% CI 1.1-15.0; data from June 5, 2021) than with any other vaccination. There was a predominance of cases in young and male patients, especially after administration of the second dose and a clustering of cases within the first week following vaccination. Outcome data indicated that patients generally recovered from symptoms quickly [3].

Recent data gathered from more than 2 million people, who received at least one COVID-19 vaccination at 40 hospitals from Washington, Oregon, Montana and California, reported an incidence rate for post-COVID-19 vaccination myocarditis of 1 per 100,000 and of post-vaccination pericarditis of 1.8 per 100,000 [4]. The study confirms that myocarditis after mRNA COVID-19 vaccination occurs preferably in younger (median age 36 years) men (75\%), soon after a median delay of 3.5 days after immunization and mostly $(80 \%)$ after the second dose, but pericarditis occurs preferably in older (median age 59 years) men (73\%) and later at a median value of 20 days after immunization with either the first $(41 \%)$ or the second dose (59\%). Hospitalization rate of myocarditis/pericarditis patients was $95 \%$ and $35 \%$, 

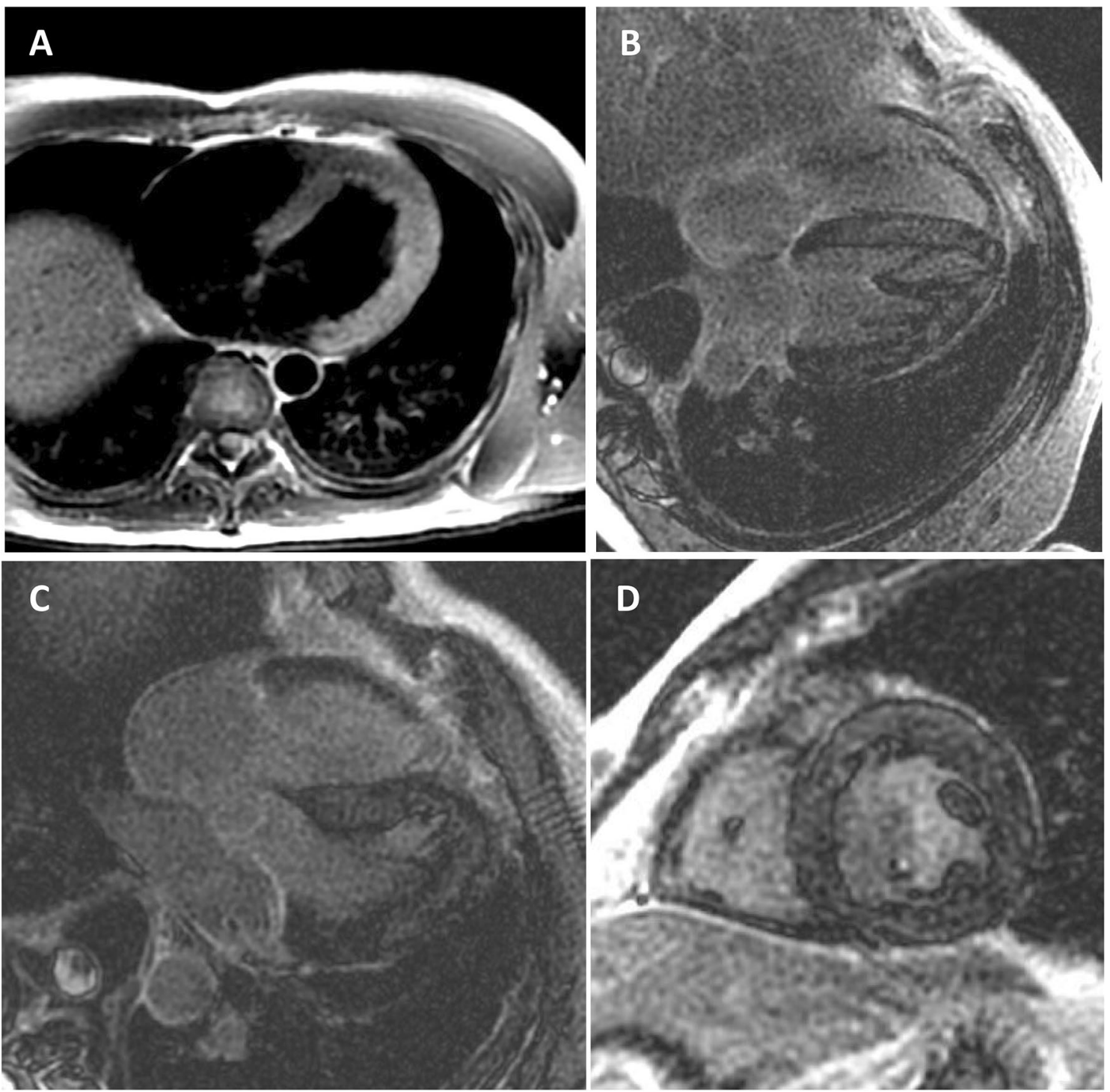

Fig. 4 Cardiac MRI study on day eight after admission. No relevant enhancement of the endo- or pericardium. No pericardial effusion. Left ventricular wall thickening with septal wall thickness reaching $16 \mathrm{~mm}$. In almost all wall segments of the left ventricle signs of acute myocarditis according to the updated Lake Louise criteria [1] were

respectively. Hospitalized patients were discharged after a median of 2 days and after one day, respectively. None of the patients died.

To the best of our knowledge, this is the first case of a biopsy-proven myocarditis after the first dose of COVID19 vaccination with an mRNA vaccine. In most reported cases of suspected post COVID-19 vaccination myocarditis, endomyocardial biopsy was not performed, but diagnosis of myocarditis was based on elevated myocardial necrosis markers and "typical" cardiac MRI findings. In the recent case series of Larson et al. [5] and Rosner et al. [6], only two patients underwent endomyocardial detected. Moderate diffuse high signal of the left ventricle on the T2w fast spin echo (FSE) fat saturation black blood sequence indicating myocardial edema (A). Partly focal to diffuse pronounced contrast enhancement on the fat saturated late gadolinium contrast enhanced sequences. Four chamber views $(\mathbf{B}, \mathbf{C})$. Short axis view (D)

biopsy-however, histology did not reveal myocarditis in either case. Although the authors explain the negative results with the sampling bias, the shortcoming of a lack of confirmation of the diagnosis of myocarditis by definite histological evidence remains.

Although we cannot definitely rule out the possibility that the myocarditis in our patient was just a coincidental association with COVID-19 vaccination, the close temporal relationship, which is also reported in other case series and the lack of a specific cardiotropic virus in the biopsies argue for a vaccine reaction.

Some points in our case deserve to be highlighted: 
As the patient developed myocarditis a few days after the first vaccination in absence of anti-SARS-CoV-2-antibodies, the pathogenesis of mRNA COVID-19 vaccine associated myocarditis does not appear to depend on anti-SARS CoV-2 spike protein antibodies. Thus, the hypothesis of crossreactivity of antibodies induced by mRNA vaccination with myocardial antigens (molecular mimicry [7]) is not corroborated by our case. Rather, the quick cardiac infiltration of immune cells after vaccination suggests that myocarditis may be caused by other mechanisms.

Unlike myocarditis associated with smallpox vaccination [8], the myocarditis following mRNA COVID-19 vaccination in our case was not an eosinophilic but a pure lymphocytic myocarditis. As acute lymphocytic myocarditis has a better prognosis with a high potential of healing under supportive and heart failure therapy compared with eosinophilic or giant-cell myocarditis [9] this might explain the more benign course of myocarditis associated with mRNA COVID-19 vaccination.

In our case, there was a discrepancy between rapid improvement of symptoms and left ventricular function despite persistent myocardial inflammation as documented by extensive late gadolinium enhancement (LGE) in the cardiac MRI study. This finding should prompt caution when deciding whether to continue drug therapy or to allow the patient to resume intense sporting activities. As both, the presence of LGE [10] as well as the detection of an inflammatory infiltrate in the endomyocardial biopsy [11] confer an increased risk for cardiac death and major adverse cardiac events, clinical decision making in these patients should not rely solely on symptom status and left ventricular ejection fraction.

Supplementary Information The online version contains supplementary material available at https://doi.org/10.1007/s00392-021-01936-6.

\section{References}

1. Ferreira VM, Schulz-Menger J, Holmvang G, Kramer Ch, Carbone I, Sechtem U et al (2018) Cardiovascular magnetic resonance in nonischemic myocardial inflammation. Expert Recommendations J Am Coll Cardiol 72:3158-3176

2. Government of Israel. Surveillance of myocarditis (inflammation of the heart muscle) cases between December 2020 and May 2021 (including) [Internet]. Jerusalem: Government of Israel; 2021 [modified 2021 Jun 02; cited 2021 Jun 10]. https://www.gov.il/ en/departments/news/01062021-03

3. Centres for Disease Control and Prevention. Clinical considerations: myocarditis and pericarditis after receipt of mRNA COVID19 vaccines among adolescents and young adults [Internet]. Atlanta, GA: Centers for Disease Control and Prevention; 2021 [cited 2021 Jun 11]. https://www.cdc.gov/vaccines/covid-19/clini cal-considerations/myocarditis.html

4. Diaz GA, Parsons GT, Gering SK, Meier AR, Hutchinson IV, Robicsek A (2021) Myocarditis and pericarditis after vaccination for COVID-19. JAMA August 04. https://doi.org/10.1001/jama. 2021.13443

5. Larson KF, Ammirati E, Adler ED, Cooper LT, Hong KN, Saponara $\mathrm{G}$ et al (2021) Myocarditis after BNT162b2 and mRNA-1273 vaccination. Circulation Jun 16. https://doi.org/10.1161/CIRCU LATIONAHA.121.055913.

6. Rosner CM, Genovese L, Tehrani BN, Atkins M, Bakhshi H, Chaudhri $\mathrm{S}$ et al (2021) Myocarditis temporally associated with COVID-19 vaccination. Circulation Jun 16. https://doi.org/10. 1161/CIRCULATIONAHA.121.055891.

7. Kanduc D (2020) From anti-SARS-CoV-2 immune responses to COVID-19 via molecular mimicry. Antibodies 9: $33 \mathrm{https} / / /$ doi. org/10.3390/antib9030033.

8. Murphy JG, Wright RS, Bruce GK, Baddour LM, Farrell MA, Edwards WD et al (2003) Eosinophilic-lymphocytic myocarditis after smallpox vaccination. Lancet 362:1378-1380

9. Tschöpe C, Ammirati E, Bozkurt B, Caforio AL, Cooper LT, Felix SB et al (2021) Myocarditis and inflammatory cardiomyopathy: current evidence and future directions. Nat Cardiol 18:169-193

10. Georgiopoulos G, Figliozzi S, Sanguineti F, Aquaro GD, di Bella G, Stamatepoulos K et al. (2021) Prognostic impact of late gadolinium enhancement by cardiovascular magnetic resonance in myocarditis. A systematic review and meta-analysis. Circ Cardiovasc Imaging 14: e011492.

11. Kindermann I, Kindermann M, Kandolf R, Klingel K, Bültmann B, Müller Th et al (2008) Predictors of outcome in patients with suspected myocarditis. Circulation 118:639-648 